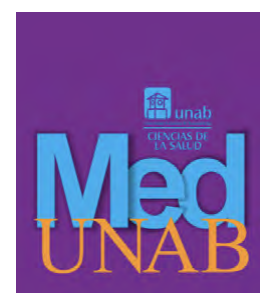

REVISTA DE LA FACULTAD

DE CIENCIAS DE LA SALUD

\title{
Caracterización de lesiones radiolúcidas del complejo maxilar-mandíbula de pacientes en un centro radiológico
}

Characterization of Radiolucent Lesions in Patients' Maxillary and Mandible Systems in a Radiology Center

Caracterização das lesões radiolúcidas do complexo maxilo mandibular de pacientes em um centro radiológico

Yuli Andreina González Moret, Odon. ${ }^{1}$ (D), Yurilú Andreina González Moret, MD., Esp. ${ }^{2}$ (iD , Yuli Nohemi Moret de Gonzalez, Odon., MSc, Esp. ${ }^{3}$ iD

1. Odontóloga. Egresada de la Universidad Central de Venezuela (UCV).

2. Médico, Especialista en Medicina Interna. Departamento de Medicina Interna. Hospital Militar Universitario Dr. Carlos Arvelo, San Martín, Venezuela.

3. Odontóloga. Magister Scientiarum en Medicina Estomatológica. Especialista en Radiología Oral y Maxilofacial. Profa. Titular de la Cátedra de Anatomía Patológica. Coordinadora de Investigación de la Facultad de Odontología de la Universidad Central de Venezuela (UCV).

Correspondencia. Yurilú Andreina González Moret. Hospital Militar Universitario "Dr. Carlos Arvelo". Departamento de Medicina Interna. Av. Principal Country Club, El Bosque, Chacao. Estado Miranda. Venezuela. Email: yuri3090@gmail.com

\section{INFORMACIÓN DEL ARTÍCULO:}

Artículo recibido: 31 de marzo de 2020

Artículo aceptado: 06 de julio de 2020

DOI: https://doi.org/10.29375/01237047.3877

Cómo citar: González-Moret YA, González Y, Moret-De González, YN. Caracterización de lesiones radiolúcidas del complejo maxilar-mandíbula de pacientes en un centro radiológico. MedUNAB. 2020;23(3): 441-449. doi: https://doi.org/10.29375/01237047.3877

\section{RESUMEN}

Introducción. Las lesiones radiolúcidas del complejo maxilar mandíbula tienen su origen en estructuras odontogénicas y no odontogénicas. El objetivo de este trabajo es describir las características de lesiones radiolúcidas del complejo maxilar-mandíbula de pacientes en un centro radiológico. Metodología. Se realizó un estudio descriptivo de corte transversal. La población está representada por 
1,000 radiografías panorámicas. La muestra quedó conformada por 105 casos seleccionados por un muestreo de conveniencia no probabilístico. Las radiografías fueron tomadas con un equipo panorámico Gendex 9,000, por un mismo operador, y fueron analizadas por un investigador calificado. Resultados. La mayoría de las lesiones radiolúcidas se ubicaron en el rango etario de 50 a 59 años $(30=28.6 \%)$, fueron del género masculino $(54=51.4 \%)$, y estuvieron ubicadas en mandíbula $(83=79.0 \%)$. Respecto a la estructura dentaria, en su mayoría las lesiones estuvieron ubicadas en la porción periapical $(65=61.9 \%)$, en relación con la forma, ésta fue predominantemente irregular $(68=64.8 \%)$, con bordes difusos $(51=48.6 \%)$ y estructura interna unilocular $(74=70.5 \%)$. En ninguno de los casos se evidenció asociación estadísticamente significativa $(\mathrm{p}>0.05)$. Discusión. La gran diversidad de diagnósticos hallados se puede explicar por las condiciones geográficas, sociales, culturales, proceso biológico de envejecimiento, y condiciones inherentes al sitio de recolección de datos. Conclusiones. La mayoría de las lesiones se localizan en mandíbula, en relación con la estructura dentaria son periapicales, presentan bordes difusos y están presentes en el grupo etario de 50 a 59 años. Las lesiones fueron en su mayoría de forma irregular bordes difusos y con estructura interna unilocular.

Palabras claves:

Odontología; Mandíbula; Maxilar; Radiografía panorámica; Diagnóstico por imagen.

\section{ABSTRACT}

Introduction. Radiolucent lesions of the maxillary and mandible system arise from odontogenic and non-odontogenic structures. This project's objective is to describe the characteristics of radiolucent lesions in patients' maxillary and mandible systems in a radiology center. Methodology. A descriptive, cross-sectional study was performed. The population is represented by 1,000 panoramic radiographs. The sample was comprised of 105 cases selected by convenience and nonprobability sampling. The radiographs were taken with a Gendex 9,000 panoramic device, by one same operator, and they were analyzed by a qualified researcher. Results. Most radiolucent lesions were located in the age group of 50 to 59 years of age $(30=28.6 \%)$, in males $(54=51.4 \%)$ and on the mandible $(83=79.0 \%)$. With respect to tooth structure, most lesions were located on the periapical portion $(65=61.9 \%)$. Regarding their form, it was predominantly irregular $(68=64.8 \%)$ with diffuse edges $(51=48.6 \%)$ and a unilocular internal structure $(74=70.5 \%)$. In no case was a statistically significant association evident ( $\mathrm{p}>0.05$ ). Discussion. The great diversity of observed diagnoses can be explained by geographical, social and cultural conditions, as well as the biological process of aging and conditions inherent to the data collection site. Conclusions. Most lesions were located on the mandible. With respect to tooth structure, they were periapical, with diffuse edges and present in the age group of 50 to 59 years of age. The lesions were mostly irregular with diffuse edges and an unilocular internal structure.

Keywords:

Dentistry; Mandible; Maxillary; Panoramic radiography; Diagnostic imaging.

\section{RESUMO}

Introdução. As lesões radiolúcidas do complexo maxilo mandibular têm sua origem em estruturas odontogênicas e não odontogênicas. O objetivo deste trabalho é descrever as características das lesões radiolúcidas do complexo maxilo mandibular de pacientes em um centro radiológico. Métodos. Realizou-se um estudo transversal e descritivo. A população é representada por 1,000 radiografias panorâmicas. A amostra foi composta por 105 casos selecionados por amostragem não probabilística por conveniência. As radiografias foram realizadas usando o aparelho panorâmico Gendex 9,000, pelo mesmo operador, e analisadas por um pesquisador qualificado. Resultados. A maioria das lesões radiolúcidas localizava-se na faixa etária de 50 a 59 anos $(30=28.6 \%)$, foram do gênero masculino $(54=51.4 \%)$ e estavam localizadas na mandíbula $(83=79.0 \%)$. Em relação à estrutura dentária, a maioria das lesões foram achadas na porção periapical $(65=61.9 \%)$; em relação à forma, esta foi predominantemente irregular $(68=64.8 \%)$, com bordas difusas $(51=48.6 \%)$ e estrutura interna unilocular $(74=70.5 \%)$. Em nenhum dos casos foi evidenciada associação estatisticamente significativa ( $p>0.05$ ). Discussão. A grande diversidade de diagnósticos achados 
pode ser explicada pelas condições geográficas, sociais, culturais, pelo processo de envelhecimento biológico e pelas condições inerentes ao local de coleta de dados. Conclusão. A maioria das lesões estavam localizadas na mandíbula. Em relação à estrutura dentária são periapicais, possuem bordas difusas e estão presentes na faixa etária de 50 a 59 anos. As lesões foram, majoritariamente, de forma irregular, bordas difusas e com estrutura interna unilocular.

Palavras-chave:

Odontologia; Mandíbula; Maxilar; Radiografia panorâmica; Diagnóstico por imagen.

\section{Introducción}

Las lesiones radiolúcidas del Complejo MaxiloMandibular, (CMM) tienen su origen en estructuras odontogénicas y no odontogénicas, con una gran variedad de apariencias tanto sólidas como quísticas y con diferentes grados de potencial destructivo (1). Las lesiones radiolúcidas del CMM se clasifican según la Organización Mundial de la Salud (OMS) 2017 en: quistes odontogénicos de origen inflamatorio (quiste radicular y quiste inflamatorio colateral), quistes odontogénicos (quiste dentígero, queratoquiste odontogénico, quiste periodontal lateral y botroídeo, quiste gingival, quiste odontogénico glandular, quiste odontogénico calcificante, y quiste odontogénico ortoqueratinizado) y finalmente los quistes no odontogénicos o del desarrollo (quiste del conducto nasopalatino) (2).

Para simplificar la frecuencia en la que aparecen se ha reportado una descripción sistemática basada en la localización anatómica, el tamaño, la forma, los bordes, la radiodensidad y el efecto sobre estructuras adyacentes (3). Dentro de esta clasificación, se hace referencia a las características de los bordes, donde se ha descrito que los bien definidos son aquellos en donde la mayoría de la periferia está bien delimitada (4), estos bordes son característicos de lesiones tipo: quiste radicular, residual, primordial, dentígero, queratoquiste, ameloblastoma, mixoma odontogénico, quiste óseo simple, granuloma eosinofílico, y granuloma central de células gigantes $(5,6)$. Los bordes mal definidos son aquellos invasivos, que no están nítidos debido a una transición gradual entre la trabécula ósea de aspecto normal y la de aspecto anormal (4). A su vez, los bordes mal definidos se han asociado a: abscesos periapicales crónicos, osteomielitis, osteonecrosis asociada a medicamentos, osteoradionecrosis, osteoblastoma, carcinoma espino celular, y metástasis entre otros $(5,6)$. Los bordes corticalizados son aquellos que poseen una línea delgada, radiopaca, bastante uniforme de hueso reactivo en la periferia de la lesión, mientras que los bordes no corticalizados carecen de este margen esclerótico (4). La radiografía panorámica es un estudio complementario para el diagnóstico de estas lesiones, ya que se puede valorar en una imagen una gran área en donde se demuestran todos los tejidos, es fácil de comprender y permite una valoración rápida de cualquier lesión en el $\mathrm{CMM}(5,6)$.

En un estudio sobre la frecuencia de hallazgos en las radiografías panorámicas de pacientes atendidos en la Universidad Santo Tomás de Colombia, se encontró que de las 766 radiografías evaluadas 457 (59.7\%) pertenecían al género femenino, que las lesiones más prevalentes se ubicaban en maxilar, siendo las radiolúcidas las más comunes con 284 (37.1\%) casos (7).

En el año 2016, Araujo et al. evaluaron lesiones del CMM y demostraron que la mandíbula fue la más afectada $(71.43 \%)$, principalmente en la región del cuerpo mandibular. Los quistes dentígeros $(100 \%)$ y los quistes residuales $(66.67 \%)$ fueron más frecuentes en la mandíbula y los quistes radiculares $(57.9 \%)$ fueron más frecuentes en el maxilar. Los ameloblastomas (100\%), el queratoquiste odontogénico $(87.5 \%)$, el odontoma compuesto (57.14\%) y los tumores odontogénicos quísticos calcificantes $(66.66 \%)$ ocurrieron con mayor frecuencia en la mandíbula (1).

Barrientos, en el año 2015, realizó un estudio descriptivo de prevalencia sobre lesiones radiolúcidas, radiopacas y mixtas que afectan el maxilar y la mandíbula en la Universidad de San Carlos en Guatemala. La muestra quedó constituida por 210 radiografías panorámicas que fueron seleccionadas de acuerdo con los criterios de exclusión e inclusión establecidos. Las variables por estudiar fueron: género, presencia de imagen, número de imágenes, maxilar y segmento implicado, área y diagnóstico diferencial. Se encontró que 130 (61.9\%) correspondían a imágenes radiolúcidas considerándolas las más frecuentes. Los diagnósticos diferenciales fueron granuloma periapical y quiste periapical, lesiones que se clasifican dentro del grupo de lesiones como radiolúcidas (8).

Naranjo y Tastets, en el año 2017, estudiaron la prevalencia de lesiones radiolúcidas mandibulares a través de radiografías panorámicas. Realizaron un estudio descriptivo, observacional y transversal. La muestra estaba constituida por 307 radiografías panorámicas. Las variables por estudiar fueron origen de la lesión, género, 
edad, ubicación general y detallada, concluyendo que las lesiones radiolúcidas de origen inflamatorio fueron más frecuentes entre 50 a 59 años (29.6\%), género masculino (15 casos 55.6\%) y localizadas en la mandíbula, en zona de molares y premolares (9).

Escobar, Romero y Aguilar, en el año 2019, estudiaron la prevalencia de lesiones maxilares de pacientes que acuden a la consulta radiológica de la Universidad Autónoma de Honduras. Realizaron un estudio de enfoque cuantitativo, alcance descriptivo, diseño transversal y observacional. La muestra quedó constituida por 86 pacientes $(26.3 \%)$ que presentaban lesiones maxilares y mandibulares, el género femenino fue el más frecuente con 49 casos $(56.9 \%)$, el grupo etario más prevalente fue el de 21 a 30 años (21 casos $24.4 \%)(10)$.

Si estas lesiones no son diagnosticadas a tiempo pueden producir secuelas en el CMM como, por ejemplo, pérdida de la función masticatoria y deformidades faciales. Por lo antes expuesto se destaca la importancia de estos estudios que permiten conocer la caracterización, ubicación y distribución de estas lesiones para facilitar su detección temprana y promover el tratamiento oportuno. Hoy en día la literatura es escasa en Venezuela lo que hace pertinente esta investigación. El objetivo de este trabajo es describir las características de lesiones radiolúcidas del complejo maxilar-mandíbula de pacientes en un centro radiológico.

\section{Metodología}

De acuerdo con el estudio planteado, la investigación realizada es de tipo descriptivo de corte transversal. Se realizó una recolección de datos directamente de la realidad donde ocurren los hechos sin manipular o controlar las variables. La población del presente estudio está representada por 1,000 radiografías panorámicas del Centro Radiológico La Villa, tomadas durante el período 2018-2019. Para cumplir con los objetivos de la presente investigación, la muestra evaluada quedó conformada por 105 casos, escogidos por un muestreo por conveniencia, intencional no probabilístico. Se realizó el cálculo de tamaño de muestra para una población total de 1,000 radiografías panorámicas, tomando un margen de error del $10 \%$ y un nivel de confianza del $95 \%$, para un tamaño de muestra de 88 radiografías panorámicas. Para la selección de la muestra se establecieron los siguientes criterios de inclusión y exclusión.

Criterios de inclusión: pacientes adultos con lesiones radiolúcidas en el complejo maxilar-mandíbula, radiografias que cumplan con los criterios técnicos exigidos.
Criterios de exclusión: radiografías que no cumplan con los criterios de calidad exigida.

La información fue recogida de una base de datos preexistente y se transcribió en una tabla de recolección de datos elaborada para tal fin. Se realizó la revisión detallada de las radiografías panorámicas procesadas por un ordenador digital, tomadas con un equipo panorámico Gendex 9,000 y por un operador calificado. Las radiografias debían cumplir con características de contraste, geometría de la imagen, nitidez y resolución. Las mismas fueron analizadas por un investigador previamente calibrado por un experto en el área. Para la adecuada clasificación y tabulación de los resultados, cada variable en estudio se codificó con anterioridad como se describe a continuación. La variable edad se dividió en 5 grupos etarios clasificados por décadas (20 a 29 años, 30 a 39 años, 40 a 49 años, 50 a 59 años y $\geq 60$ años), género (femenino y masculino), afectación (maxilar y/o mandíbula), relación con estructura dentaria (periapical, interradicular, pericoronaria y no asociada al diente), forma (irregular, redondeada, ovalada y piriforme), borde (difuso, definido, corticalizado y no corticalizado) y la estructura interna de la imagen (unilocular y multilocular) según lo reportan Langlais y Kasle en 1987 (11).

La información de las variables recolectadas fue tratada desde el punto de vista descriptivo, realizando tablas en el programa Microsoft Office Excel 2014 y realizando el análisis de las variables en el programa SPSS Versión 24. Se realizó la prueba de Kolmogorov-Smirnov para verificar la distribución normal de las variables. Las variables nominales se describieron como frecuencias absolutas y relativas. La prueba de Chi cuadrado (Chi2) fue calculada para determinar asociación entre variables cualitativas. Para todas las pruebas inferenciales se consideró el valor de $\mathrm{p}$ de dos colas significativas, menor o igual a 0.05 . Se utilizaron gráficas, según el caso, como elementos de apoyo para el análisis y la discusión de los resultados.

Para la realización del estudio se contó con un permiso otorgado por la dirección del Centro Radiológico La Villa en el cual se encontraba la base de datos de la investigación. Los datos personales de los pacientes que conformaron la muestra se mantuvieron confidenciales haciendo uso de una identificación numérica. El consentimiento informado del paciente forma parte del archivo, donde se les explica el procedimiento de la toma de la radiografía y a la vez se les informa que la misma puede ser usada con fines docentes y de investigación. Se cumplieron las normativas de la declaración de Helsinki 2,000 (autonomía, beneficencia, no maleficencia y justicia). Se solicitó el aval del comité de Bioética del Centro Radiológico La Villa el cual fue otorgado bajo el $N^{\circ}$ CBCRV 4-2019. El comité de bioética 
de la institución se rige por las normas de buena práctica clínica para ensayos clínicos emitida por el Ministerio del Poder Popular para la Salud de fecha 8 de julio de 2013. Así, el estudio se clasifica como una investigación sin riesgo.

\section{Resultados}

En total se evaluaron 1,000 ejemplares de radiografías panorámicas, de los cuales 137 fueron excluidos por ser pacientes pediátricos y 758 tampoco participaron en vista de que no presentaban las características imagenológicas en estudio, obteniendo una muestra total (n) de 105 radiografías panorámicas.
Con respecto a las características y distribución de las imágenes radiolúcidas que afectan el complejo maxilarmandíbula, se evidenció que de la muestra total $(\mathrm{n}=105)$, la mayoría se encontraba dentro del rango etario entre 50 a 59 años $(30=28.6 \%)$ y en su minoría $(7=6.7 \%)$ entre 20 y 29 años con un promedio de edad de 49.31 años. Con respecto al género, $(54=51.4 \%)$ correspondían al género masculino, la mayoría de las lesiones $(83=79.0 \%)$ se ubicaban en la mandíbula $\mathrm{y}$, con respecto a la relación con la estructura dentaria, el mayor número $(65=61.9 \%)$ estaba ubicado en la porción periapical dentaria. Por su parte, al evaluar la forma de la lesión, la mayoría eran irregulares $(68=64.8 \%)$. Los bordes eran difusos $(51=48.6 \%)$ y, en su minoría, $(2=1.9 \%)$ no corticalizados. Finalmente, la estructura interna de la imagen evidenció que fueron uniloculares en su mayoría $(74=70.5 \%)$. Todas las variables presentaron una distribución normal $(\mathrm{p}>0.05)$ (tabla 1).

Tabla 1. Características de imágenes radiolúcidas que afectan el complejo maxilar-mandíbula de pacientes que acuden al Centro Radiológico La Villa.

Características

Muestra evaluada: $\mathbf{n}$

Grupo etario (\%)

20-29 años

30-39 años

40-49 años

50-59 años

+66 años
Valor de p

105

17 (16.2)

$25(23.8)$

$30(28.6)$

$26(24.8)$

Afectación maxilar y /o mandíbula: n (\%)

Maxilar

Mandíbula
$22(21.0)$

0.486

$83(79.0)$

Relación con estructura dentaria: $n$ (\%)

$\begin{array}{cc}\text { Periapical } & 65(61.9) \\ \text { Interradicular } & 19(18.1) \\ \text { Pericoronaria } & 0(0)\end{array}$

No relacionada al diente

$21(20.0)$

Forma de la imagen

$\begin{array}{cc}\text { Irregular } & 68(64.8) \\ \text { Ovalada } & 4(3.8) \\ \text { Redonda } & 33(31.4) \\ \text { Piriforme } & 0(0)\end{array}$




\section{Borde de la imagen}

Difuso

No corticalizado

Definido

Corticalizado

\section{Estructura Interna}

$$
\begin{gathered}
\text { Multilocular } \\
\text { Unilocular }
\end{gathered}
$$

$$
51(48.6)
$$

$31(29.5)$

Fuente: elaborada por los autores

Al analizar la asociación entre las imágenes radiolúcidas que afectan el CMM según el grupo etario, se evidenció que la mayoría de las lesiones $(23=27.7 \%)$ se encontraban en la mandíbula de los pacientes entre 50 y 59 años, no evidenciando una asociación estadísticamente significativa entre dichas variables (Chi2 $=1.63, \mathrm{p}=0.802)$ ( figura 1).

Figura 1. Distibución de lesiones radiolúcidas según el grupo etario y la afectación del complejo maxilomandibular (CMM). Significancia (bilateral): 0.802

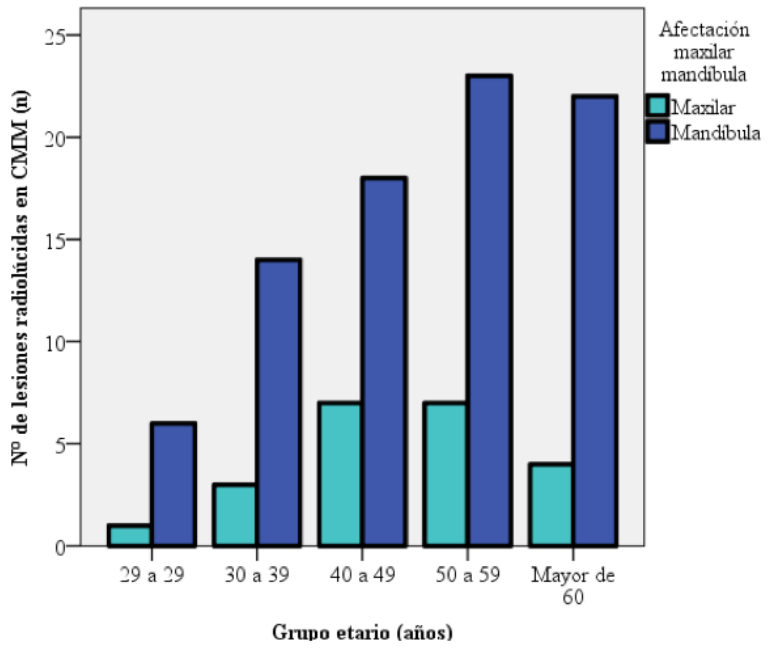

Fuente: elaboración propia

Con respecto a la distribución de las lesiones radiolúcidas que afectan el CMM, según el grupo etario y los bordes de la lesión, se observó como la mayoría de las imágenes patológicas, se encontraron en el grupo mayor de 60 años presentando bordes difusos $(17=33.3 \%)$. Este resultado no demostró asociación significativa estadísticamente (Chi2= 14.765, $\mathrm{p}=0.255$ ) (figura 2).
Figura 2. Distribución de lesiones radiolúcidas según el grupo etario y los bordes de la lesión. Significancia (bilateral): $\mathrm{p}=0.255$

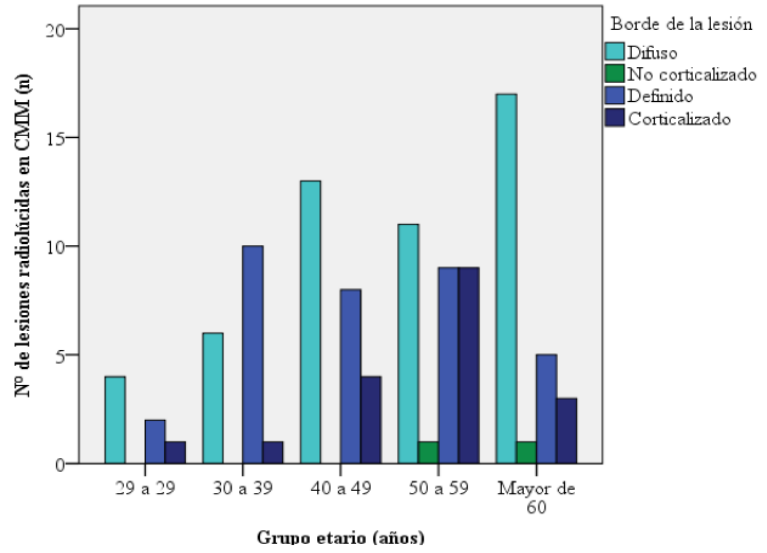

Fuente: elaboración propia

Por su parte, la distribución de las lesiones radiolúcidas que afectan el CMM con respecto al género, demostró que la mayoría eran de género masculino y se encontraban en la mandíbula $(44=53.0 \%)$. No se encontró asociación estadísticamente significativa entre dichas variables (Chi2 $=0.398, \mathrm{p}=0.528)$ (ver anexos figura 3).

Figura 3. Distribución de lesiones radiolúcidas que afectan el complejo máxilo-mandibular

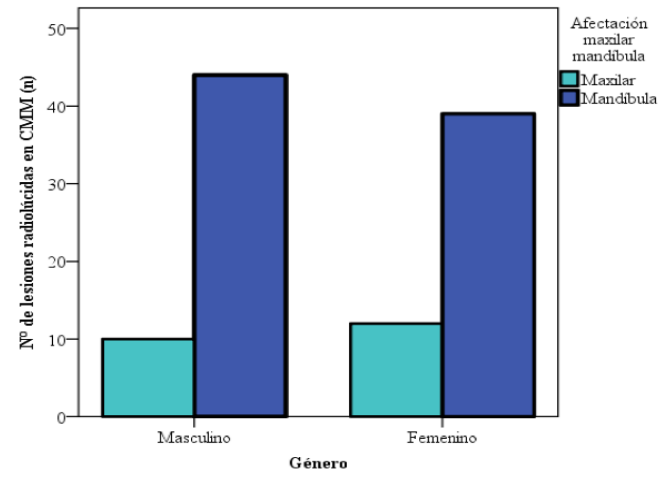

Fuente: elaboración propia. Significancia (bilateral): $\mathrm{p}=0.528$ 
En el mismo orden de ideas, se evidenció cómo las lesiones radiolúcidas que afectan el CMM, de acuerdo con su relación con la estructura dentaria, eran en su mayoría periapicales ubicándose a nivel mandibular $(50=60.2 \%)$. No hubo asociación significativa entre estas variables (Chi2 $=0.741, p=0.690)$ (ver anexos figura 4).

Figura 4. Distribución de lesiones radiolúcidas de acuerdo con la relación con la estructura dentaria.

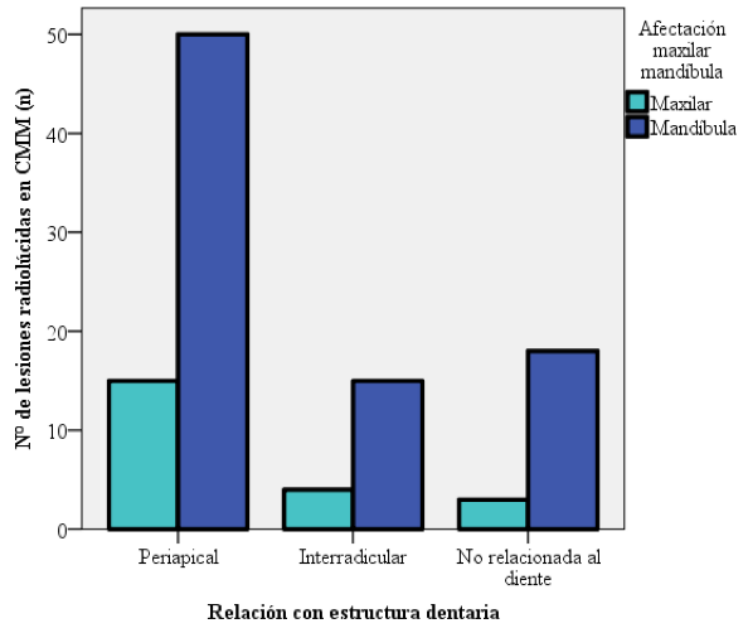

Fuente: elaboración propia. Significancia (bilateral): $\mathrm{p}=0.690$

Finalmente, con respecto a la distribución de las lesiones radiolúcidas que afectan el CMM de acuerdo con la forma, bordes y estructura interna de dichas lesiones, se determinó que: la mayoría de las lesiones tenían forma irregular $(56=67.5 \%)$, bordes difusos $(41=49.4 \%)$ y con estructura interna unilocular $(59=71.1 \%)$, todas ubicadas a nivel mandibular. En ningún caso se evidenció asociación estadísticamente significativa entre las variables en estudio (Chi2: $3.251 \mathrm{p}=0.197$; Chi2 $=1.095 \mathrm{p}=0.778$; Chi2 $=0.070 \mathrm{p}=0.791$ respectivamente) (figuras 5, 6 y 7).

Figura 5. Distribución de lesiones radiolúcidas de acuerdo con la forma de la lesión.

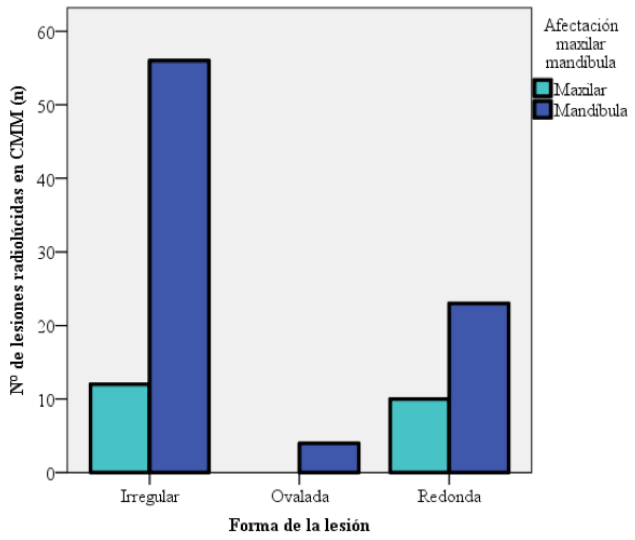

Fuente: elaboración propia. Significancia (bilateral): $\mathrm{p}=0.197$
Figura 6. Distribución de lesiones radiolúcidas que afectan el complejo máxilo-mandibular de acuerdo con los bordes de la lesión. Significancia (bilateral): $\mathrm{p}=0.778$.

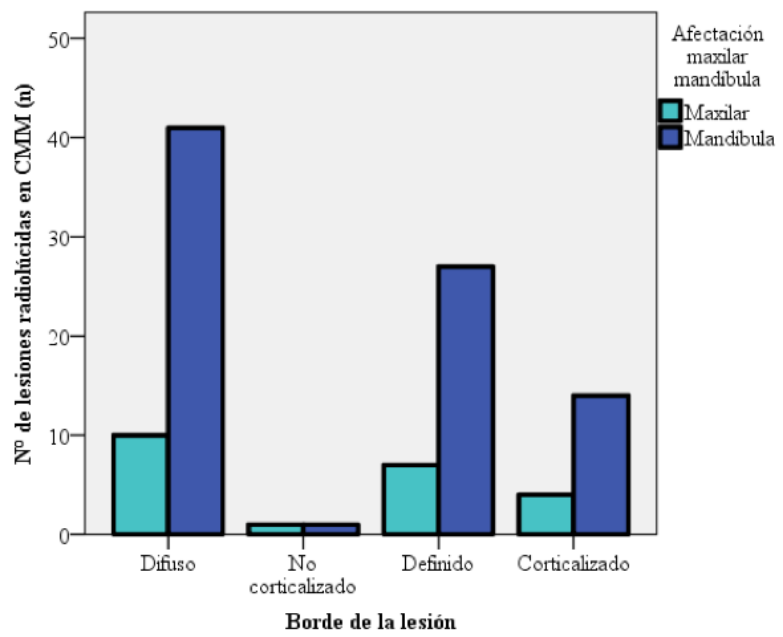

Fuente: elaboración propia.

Figura 7. Distribución de lesiones radiolúcidas que afectan el complejo máxilo-mandibular según la estructura interna de la lesión. Significancia (bilateral): $\mathbf{p = 0 . 7 9 1 . ~}$

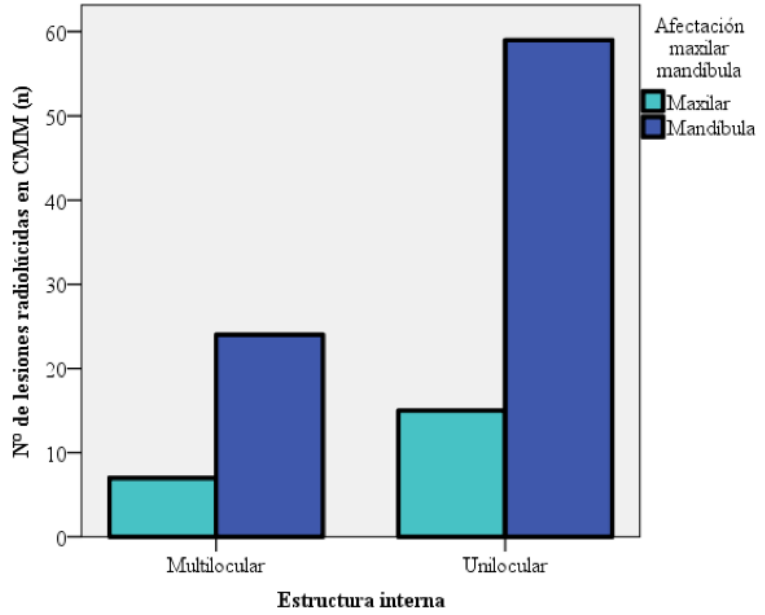

Fuente: elaboración propia.

\section{Discusión}

Al describir la caracterización de lesiones radiolúcidas con respecto al CMM, se evidenció que la mayoría se encontraba dentro del rango etario entre 50 a 59 años $(30=28.6 \%)$ con un promedio de edad de 49.31 años. Este hallazgo contrasta con los estudios de Avelar et al. en 2008 , donde el promedio alcanza tan solo los 30.7 años, con el de Thiers et al. en 2008 donde el promedio de edad fue de 35.7 años y con el de Escobar et al. en 2019, donde 
el rango de edad fue de 21 a 30 años $(10,12,13)$. En esta investigación los resultados demuestran que las lesiones radiolúcidas del CMM son más frecuentes en la población adulta, hecho que puede esta relacionado directamente con el proceso biológico de envejecimiento como reporta Castellanos en 2008, quien señala que este proceso origina una serie de cambios graduales irreversibles y acumulativos que hacen al tejido óseo más vulnerable a agentes traumáticos e infecciosos (14).

Con respecto al género, se evidenció en los resultados que existe predominio por el masculino $(54=51.4 \%)$, coincidiendo con otros estudios como Naranjo et al en el año 2017 y Thiers et al. en el 2008. Puede sumarse a este análisis que existen diferencias biológicas, culturales y sociales entre ambos sexos que hacen a los hombres más expuestos a agresiones y factores de riesgo ambientales $\mathrm{y}$ a las mujeres menos susceptibles genéticamente al desarrollo de lesiones en maxilares $(9,13)$.

Sobre el grado de afectación se encontró que la mayoría de las lesiones se ubicaron en la mandíbula $(83=79.0 \%)$, hecho que concuerda con el estudio de Toro y Sanchez en el año 2009 donde se observa que el lado más comprometido de la mandíbula fue el izquierdo, con un $52 \%$ de los casos $(n=26)$, seguido por el derecho, con un $42 \%$ de los casos $(\mathrm{n}=21)$, y finalmente la línea media con un $6 \%$ de los casos $(n=3)(15)$. Con respecto a la relación con la estructura dentaria, el mayor número estaba ubicado en la porción periapical dentaria $(65=61.9 \%)$, resultado que puede predecir una relación con lesiones de tipo odontogénicas de origen inflamatorio, tales como abscesos, granulomas y quistes periapicales. Los bordes de las lesiones fueron difusos $(51=48.6 \%)$, lo que podría indicar una alta frecuencia de lesiones en el CMM de tipo odontogénicas inflamatorias como abscesos periapicales, osteomielitis, osteonecrosis, osteoradionecrosis, así como lesiones tumorales tipo osteoblastomas, carcinomas y metástasis $(9,16-21)$.

Se puede deducir que la variabilidad en los resultados de frecuencia y características de las lesiones radiolúcidas del CMM posiblemente está determinada por condiciones geográficas, sociales, culturales e inherentes al sitio de recolección de datos (15), como lo demuestra la información recolectada de esta investigación.

Finalmente, la radiografía panorámica es una herramienta útil en el descubrimiento de diversas patologías del CMM. Un estudio en México realizado por Leyva y Vargas en el 2010, donde se analizaron imágenes panorámicas, se demostró que un $28.4 \%$ del total de la muestra, presentó un hallazgo patológico incidental (22). Otro estudio realizado por Jiménez et al en el año 2017 encontró 387 hallazgos radiográficos en pacientes adultos jóvenes, donde se destaca la importancia de la radiografía panorámica para detectar anomalías óseas y demuestra su utilidad como examen complementario de diagnóstico y tratamiento (22-24). Se puede considerar como limitación de esta investigación el tamaño de la muestra que no permite generalizar los resultados.

\section{Conclusiones}

Las características socio demográficas que describen a las imágenes radiolúcidas que comprometen el CMM están en un rango etario de 50 a 59 años $(30$ casos $=28.6 \%)$ y predominantemente en el género masculino $(54$ casos $=$ $51.4 \%)$.

Por su relación con la estructura dentaria, la mayoría de las lesiones eran periapicales, estaban localizadas en mandíbula ( 50 casos $=60.2 \%$ ), presentaban bordes difusos y estaban ubicadas en el grupo etario entre 50 a 59 años $(17$ casos $=33.3 \%)$. Con respecto a la forma, bordes y estructura interna, estas lesiones fueron en su mayoría de forma irregular $(56$ casos $=67.5 \%)$, bordes difusos $(41$ casos $=49.4 \%$ ) y con estructura interna unilocular (59 casos $=71.1 \%$ ) en todos los casos ubicados en mandíbula .

\section{Declaración de conflictos de interés}

Los autores no declaran ningún tipo de conflicto de interés.

\section{Financiamiento}

Los gastos concernientes y derivados del estudio fueron cubiertos por los autores.

\section{Referencias}

1. Araujo JP, Lemos CA, Miniello TG, Alves FA. The relevance of clinical and radiographic features of jaw lesions: A prospective study. Braz Oral Res; 2016;30(1):1-9. doi: https://doi.org/10.1590/18073107BOR-2016.vol30.0096

2. El-Naggar AK, Chan JKC, Grandis JR, Takata T, Slootweg PJ. (4th ed.). WHO Classification of Head and Neck Tumours. Berlín: International Agency for Research on Cancer, 2017. 347 p.

3. Whaites E. (4ta ed.). Fundamentos de Radiología dental. España: Elsevier Masson, 2008. 473 p.

4. White SC. (4ta ed.). Radiología Oral Principios e Interpretación. Madrid: Elsevier Science, 2002. 672 p. 
Oral Radiol Endod; 2008;105:79 - 85. doi: https://doi. org/10.1016/j.tripleo.2007.01.037

15. Toro J, Sánchez M. Inducción a la Ciencia y la Tecnología y la Innovación. UASLP; 2016;4(7):418421.

16. Avril L, Lombardi T, Ailianou A, Burkhardt K, Varoquaux A, Scolozzi P, et al. Radiolucent lesions of the mandible: A pattern-based approach to diagnosis. Insights Imaging; 2014;5(1):85-101. doi: https://doi. org/10.1007/s13244-013-0298-9

17. Herrera A, Díaz A, Herrera F, Fang L. Osteorradionecrosis como secuela de la radioterapia. Av Odontoestomatol; 2012;28(4):175-180. doi: https://doi.org/10.4321/S0213-12852012000400002

18. Morelos E, Trujillo JJ. Osteoblastoma maxilar. Reporte de un caso y revisión de la literatura. Rev Mex Ciru Buc Maxilofac; 2013;9:49-58.

19. Schott Börger S, Holmberg Pavez M. Revisión clínica y radiológica de la literatura de hipótesis diagnósticas y radiológicas, en relación a presentación preliminar de caso clínico. Clinical and radiological review of literature of diagnostic radiological hypothesis regarding. Rev Dent Chile; 2014;105(3):24-6.

20. Dunfee BL, Gohel A, Pistey R, Sakai O. Radiologic and Pathologic Characteristics of Benign and Malignant Lesions of the mandibule. RadioGraphics; 2006;26(6):1751-69. doi: https://doi.org/10.1148/ rg.266055189

21. Delbalso A. Lesions of the Jaws. Seminars in Ultrasound, CT, and MRI; 1995;16(6):487-512. doi: https://doi.org/10.1016/S0887-2171(06)80022-3

22. Leyva JL, Vargas M de la L. Hallazgos incidentales en radiografías panorámicas previas al tratamiento de ortodoncia. Acta Odontol Venez; 2011;49(3):1-9.

23. Jiménez J, Herrera J, Jiménez J, Pérez E, Murillo J. Hallazgos incidentales en ortopantomografías maxilomandibulares de pacientes adultos jóvenes. ADM; 2017;74(1):25-31.

24. Cabbar F, Cagri M, Duygu G, Tekin H, Ozcakir C. Evaluation of significant radiographic findings and their impact on the oral health-related quality of life of patients with complete dentures. Int J Prosthodont; 2018;31(6):594-600. doi: https://doi.org/10.11607/ iip. 5717 